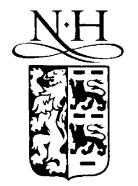

ELSEVIER

\title{
Excitation spectra of free fullerene clusters
}

\author{
Oleg Kidun *, Jamal Berakdar
}

Max-Planck Institut fuer Mikrostrukturphysik, Weinberg 2, D06120 Halle/Saale, Germany

\begin{abstract}
In this work we study the single electron emission from fullerene clusters upon the impact of low-energy electrons. The calculations of the quantum ground states of the fullerene are performed within the Hartree-Fock and the jellium shell model. The interaction between the incoming projectile and knocked out electrons is described on the basis of random phase approximation with exchange, which leads to the concept of screening of the inter-electronic interaction. We compare the results of the calculations with available experimental data for the ionization of $\mathrm{C}_{60}$ by electron impact and show that neglect of polarization leads to results at variance with experimental finding. (C) 2002 Elsevier Science B.V. All rights reserved.
\end{abstract}

Keywords: Jellium models; Many body and quasi-particle theories; Electron density, excitation spectra calculations; Electron bombardment; Electron emission; Electron-solid interactions

The aim of this work is the investigation of the single ionization of the fullerene cluster from its (single-particle) ground state $\left|\phi_{2 j}\right\rangle$ upon the impact of an electron with wave vector $\boldsymbol{k}_{0}$. In the final channel two electrons recede from the residual cluster to emerge with asymptotic momenta $\boldsymbol{k}_{1}$ and $\boldsymbol{k}_{2}$. The transition amplitude for such a reaction is given by

$T\left(\boldsymbol{k}_{0}, \boldsymbol{k}_{1}, \boldsymbol{k}_{2}, \phi_{v}\right)=E_{0}\left\langle\boldsymbol{k}_{1} \boldsymbol{k}_{2}\left|V_{12} \Pi\right| \phi_{2 j} \boldsymbol{k}_{0}\right\rangle$

$E_{0}$ is the total kinetic energy of the two electrons and $\Pi=G_{0}+G_{0} V \Pi$ is the total Green operator of the projectile-cluster system with the total potential $V$. The interaction between the projectile and the knocked-out electron is designated by $V_{12}$. Here we report on the calculation of the first-order term of Eq. (1) as well as of the next terms due to the electron-hole excitations. In the presence of the

\footnotetext{
${ }^{*}$ Corresponding author. Tel.: +49-345-5582613; fax: +49345-5511223.

E-mail address: okidun@mpi-halle.de (O. Kidun).
}

external electron with momentum $\boldsymbol{k}_{0}$ the self-consistent cluster potential changes. Taking into account the polarization of the electronic cloud, we write the amplitude of the process as a sum of two terms. The "direct" one corresponds to the excitation of the cluster's electron labeled by index " 2 " from the $j$ th bound state $\phi_{2 j}$ to the continuum state with the asymptotic momentum $\boldsymbol{k}_{2}$. The second "correlation" term describes correction, which appears from electron-hole excitations. Thus, in the random phase approximation with exchange (RPAE) the matrix element reads

$$
\begin{aligned}
T \sim & \left\langle\boldsymbol{k}_{1} \boldsymbol{k}_{2}\left|V_{\mathrm{eff}}\right| \phi_{2 j} \boldsymbol{k}_{0}\right\rangle \\
= & \left\langle\boldsymbol{k}_{1} \boldsymbol{k}_{2}\left|V_{12}\right| \phi_{2 j} \boldsymbol{k}_{0}\right\rangle \\
& +\sum_{\varepsilon_{\alpha} \leqslant \varepsilon_{\mathrm{F}}<\varepsilon_{\beta}}\left(\frac{\left\langle\boldsymbol{\alpha} \boldsymbol{k}_{2}\left|V_{\mathrm{eff}}\right| \phi_{2 j} \boldsymbol{\beta}\right\rangle\left\langle\boldsymbol{\beta} \boldsymbol{k}_{1}\left|\frac{1}{r_{13}}\right| \boldsymbol{k}_{0} \boldsymbol{\alpha}\right\rangle}{\varepsilon_{0}-\left(\varepsilon_{\alpha}-\varepsilon_{\beta}-\mathrm{i} \delta\right)}\right. \\
& \left.-\frac{\left\langle\boldsymbol{\beta} \boldsymbol{k}_{2}\left|V_{\mathrm{eff}}\right| \phi_{2 j} \boldsymbol{\alpha}\right\rangle\left\langle\boldsymbol{\alpha} \boldsymbol{k}_{1}\left|\frac{1}{r_{13}}\right| \boldsymbol{k}_{0} \boldsymbol{\beta}\right\rangle}{\varepsilon_{0}+\left(\varepsilon_{\alpha}-\varepsilon_{\beta}-\mathrm{i} \delta\right)}\right)
\end{aligned}
$$


$V_{\text {eff }}$ being an effective interelectron interaction, $1 / r_{13}$-bare Coulomb between impact and intermediate electrons; $\alpha, \beta$ are the intermediate electron's and hole's states with the energies $\varepsilon_{\alpha}, \varepsilon_{\beta} ; \varepsilon_{0}$ and $\varepsilon_{\mathrm{F}}$ are the initial energy of the projectile and the Fermi energy respectively. The different terms in the sum describe electron-hole excitation and deexcitation.

The differential electron-impact ionization crosssection is to be summed over ionization events from all the initial bound states $\phi_{2 j}$ accessible within the energy conservation, i.e.

$$
\begin{aligned}
\frac{\mathrm{d}^{6} \sigma}{\mathrm{d}^{3} \mathbf{k}_{1} \mathrm{~d}^{3} \mathbf{k}_{2}}= & \frac{(2 \pi)^{4}}{\left|k_{0}\right|} \sum_{j}\left|T\left(\mathbf{k}_{0}, \mathbf{k}_{1}, \mathbf{k}_{2}, \phi_{2 j}\right)\right|^{2} \\
& \times \delta\left(\varepsilon_{0}-\left(\epsilon_{j}^{\text {ion }}+k_{1}^{2} / 2+k_{2}^{2} / 2\right)\right)
\end{aligned}
$$

The quantum states of the fullerene cluster are constructed within the Hartree-Fock approximation and within the spherical jellium model. The potential of $\mathrm{C}_{60}$, which is a superposition of atomic potentials, is replaced by a potential of a fullerene shell. The latter is formed by delocalized valence electrons of carbon atoms and is modelled by a potential well: $V(r)=V_{0}$ within the region $R-\Delta<$ $r<R+\Delta$, and $V=0$ elsewhere. Here $R \approx 6.7 a_{0}$ is the radius of the fullerene, the thickness of the shell is $2 \Delta \approx 2 a_{0}, a_{0}$ being the Bohr radius. The height of the well was chosen such that the experimental value of the electron affinity of $\mathrm{C}_{60}$ and the number of valence electrons are correctly reproduced.

Numerical calculation of the Hartree-Fock states is based on the non-local variable phase approach [1-3]. This method allows to find the eigen functions and eigen values through the scattering phase function and through the poles of the scattering amplitude in the complex plane of the wave vector of the particle. Such a choice of numerical realization of the self-consistent procedure allows to accelerate its convergency significantly.

For this purpose we look at the scattering of the spinless particle with the energy $E=k^{2}$ and orbital momentum $\ell$ in the nonlocal potential $V\left(r, r^{\prime}\right)$ :

$$
\frac{\mathrm{d}^{2}}{\mathrm{~d} r^{2}} u_{\ell}(r)+\left(k^{2}-\frac{\ell(\ell+1)}{r^{2}}\right) u_{\ell}(r)=\int_{0}^{\infty} \mathrm{d} r^{\prime} V_{\ell}\left(r, r^{\prime}\right) u_{\ell}\left(r^{\prime}\right)
$$

After replacement of the radial part $u_{\ell}(r)$ of the wave function by the partial amplitude and phase functions $\alpha_{\ell}(r)$ and $\delta_{\ell}(r)$ :

$$
\begin{aligned}
& u_{\ell}(r)=\alpha_{\ell}(r) \cdot F\left(\delta_{\ell}(r)\right) \\
& \mathrm{d} u_{\ell}(r)=\alpha_{\ell}(r) \cdot \mathrm{d} G\left(\delta_{\ell}(r)\right)
\end{aligned}
$$

where

$$
\begin{aligned}
& F\left(\delta_{\ell}(r)\right)=\cos \delta_{\ell}(r) j_{\ell}(k r)-\sin \delta_{\ell}(r) n_{\ell}(k r) \\
& \mathrm{d} G\left(\delta_{\ell}(r)\right)=\cos \delta_{\ell}(r) \mathrm{d} j_{\ell}(k r)-\sin \delta_{\ell}(r) \mathrm{d} n_{\ell}(k r)
\end{aligned}
$$

the Schrödinger second order differential (4) transforms to the system of the first order Riccati-type equations:

$$
\begin{aligned}
\frac{\mathrm{d}}{\mathrm{d} r} \delta_{\ell}(r)= & \left(-\frac{2}{k}\right) F^{2}\left(\delta_{\ell}(r)\right) \int_{0}^{r} \mathrm{~d} r^{\prime} V\left(r, r^{\prime}\right) \\
& \times \exp \left[-\int_{r^{\prime}}^{r} \frac{\mathrm{d} G\left(\delta_{\ell}\left(r^{\prime \prime}\right)\right)}{F\left(\delta_{\ell}\left(r^{\prime \prime}\right)\right)}\right] \\
\frac{\mathrm{d}}{\mathrm{d} r} \alpha_{\ell}(r)= & \alpha_{\ell}(r) \frac{\sin \delta_{\ell}(r) \cdot j_{\ell}(k r)+\cos \delta_{\ell}(r) \cdot n_{\ell}(k r)}{F\left(\delta_{\ell}(r)\right)} \\
& \times \frac{\mathrm{d}}{\mathrm{d} r} \delta_{\ell}(r)
\end{aligned}
$$

$j_{\ell}(k r), n_{\ell}(k r)$ - Riccati-Bessel functions are regular and irregular solutions of the free Schrödinger equation. For the local potential $V(r), \delta_{\ell(r)}$ and $\alpha_{\ell(r)}$ have clear physical interpretation: they are the asymptotic scattering phase and the asymptotic amplitude of the wave function built for cutoff at $r$ potential.

The eigen energies are calculated by finding the poles of the partial scattering amplitude in the imaginary half-axis of the wave vector $k=\mathrm{i} \kappa$, $\kappa \in \Re$. It is related to the scattering phase $f_{\ell}=(1 / k) \mathrm{e}^{\mathrm{i} \delta_{\ell}} \sin \delta_{\ell}$. After some algebra, Volterratype equation for $f_{\ell}$, corresponding to the system (6), can be derived

$$
\begin{aligned}
& \frac{\mathrm{d}\left(\mathrm{i} \cdot f_{\ell}(r)\right)}{\mathrm{d} r} \\
& =\left(-\frac{2}{\kappa}\right) \frac{1}{\beta^{2}} F^{2}\left(f_{\ell}(r)\right) \int_{0}^{r} \mathrm{~d} r^{\prime} V_{\ell}\left(r, r^{\prime}\right) \\
& \quad \times \exp \left\{\int_{r^{\prime}}^{r} \frac{\mathrm{i} \cdot f_{\ell}\left(r^{\prime \prime}\right) \cdot \mathrm{d} q_{\ell}\left(\kappa r^{\prime \prime}\right)+\beta^{2} \mathrm{~d} p_{\ell}(\kappa r)}{F\left(f_{\ell}\left(r^{\prime \prime}\right)\right)}\right\}
\end{aligned}
$$


$F\left(f_{\ell}(r)\right)=\mathrm{i} \cdot f_{\ell}(r) \cdot q_{\ell}(\kappa r)+\beta^{2} p_{\ell}(\kappa r)$

where $p_{\ell}$ and $q_{\ell}$ are modified Riccati-Bessel functions of the real argument $\kappa r ; \beta=(\mathrm{i})^{\ell+1}$. Initial condition for the integration is $f_{\ell}(r=0)=0$. The condition for the bound state is condition for the poles of $f_{\ell}(r \rightarrow \infty)$. In practical calculation the regularization of this equation can be made in two different ways: by the replacement $\mathrm{i} \cdot f_{\ell}=$ $1 / \phi$ or by the replacement $\mathrm{i} \cdot f_{\ell}=\tan \gamma$. Then the eigen state exists at zeros of $\phi$ or for $\gamma=$ $(2 n+1) \pi / 2, n \in \mathscr{Z}$. In this work we used the regularization of the first kind.

Due to the spherical symmetry the HartreeFock total wave function was constructed from $2(2 \ell+1)$ degenerate single-electron orbitals. The latter form several sub-bands characterized by different number of nodes of the radial wave functions. The lowest sub-band orbitals with the orbital momenta $0 \leqslant \ell \leqslant 7$ do not have nodes on radial axis, the next ones $(0 \leqslant \ell \leqslant 5)$ have one node, and highest sub-bands occupied orbitals (with $0 \leqslant \ell \leqslant 3$ and $0 \leqslant \ell \leqslant 2$ ) - two and three nodes respectively. All shells are closed and the total number of the electrons is equal to 240 .

To get a qualitative insight into the role of screening for the shape of the cross section we compare our RPAE calculations with the calculations in intuitively transparent approximation: Due to the presence of a large number of mobile electrons on the surface of the fullerene the interaction of the incident electron with the cluster electrons is screened. A simple model to account for this effect is the Thomas-Fermi approach which yields a local screening of the electronelectron interaction described by the one-parameter Yukawa potential: $V\left(r_{12}\right)=\exp \left(-\lambda r_{12}\right) / r_{12}$, where $\lambda$ is the inverse screening length. The results of our calculations are presented in Fig. 1a, where the total cross section of the process $\sigma\left(\varepsilon_{0}\right)$ is given as a function of the impact energy. Dashed curves correspond to different values of the inverse screening length, solid curve is the RPAE result.

Despite their conceptual simplicity, these models of electronic correlation yield encouraging results. We compare them with available experimental data [4] together with the calculation of work [5], where the bare Coulomb interaction was taken into ac- (a)

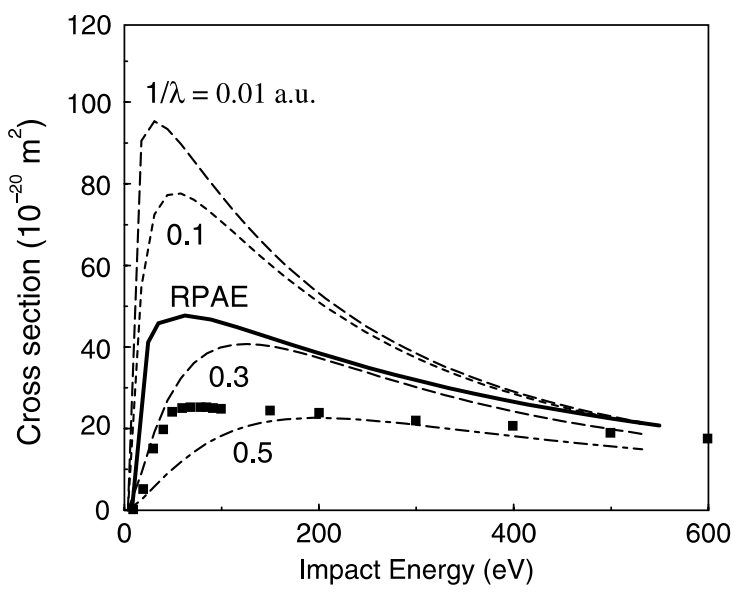

(b)

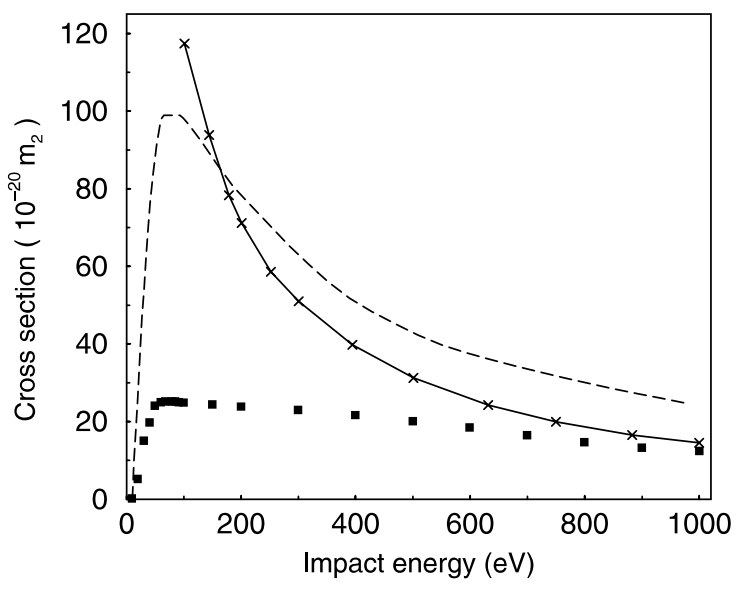

Fig. 1. Integrated electron-impact single ionization cross section of $\mathrm{C}_{60}$ as a function of the projectile energy: (a) Present calculations in Thomas-Fermi model (dashed curves) with different values of the inverse screening length and in the RPAE (solid curve). (b) Solid line: calculation in the plane wave Born approximation for the jellium-shell model [5]; dashed line: result of semi-empirical model calculation [6]; (a) and (b) symbols: experimental cross section for production of $\mathrm{C}_{60}^{+}$ions [4].

count and the $(\mathrm{e}, 2 \mathrm{e})$ total cross section was calculated in the plane wave Born approximation, and with the semi-empirical model calculation [6] (see Fig. 1b). When contrasted with calculations that neglect screening effect, the account of it results in a flattening of the total ionization cross section at lower impact energies. This effect can be traced back to the fact that for the unscreened Coulomb 
interaction the main contribution to the cross sections originates from far collisions, whereas in case of screening there is a cut-off impact parameter beyond which the contribution of collisions to the cross section diminishes.

\section{References}

[1] F. Calogero, Nuovo Cimento 27 (1) (1963) 261; F. Calogero, Nuovo Cimento 33 (2) (1964) 352.
[2] V. Babikov, Method of the phase functions in quantum mechanics. Moskow, Nauka, 1971.

[3] O. Kidun, J. Berakdar, in: Many-Particle Spectroscopy of Atoms, Molecules, Clusters and Surfaces, 2001, pp. 395404.

[4] V. Foltin, M. Foltin, S. Matt, P. Scheier, K. Becker, H. Deutsch, T.D. Mark, Chem. Phys. Lett. 289 (1-2) (1998) 181-188.

[5] S. Keller, E. Engel, Chem. Phys. Lett. 299 (2) (1999) 165170.

[6] H. Deutsch, K. Becker, J. Pittner, V. Boniacic-Koutecy, S. Matt, T.D. Mark, J. Phys. B 29 (1996) 5175. 